\title{
Ein Blick auf Geschichte und Bedeutung der Deutschen Statistischen Gesellschaft
}

\author{
Ansprache anlässlich des 100-jährigen Bestehens der Deutschen \\ Statistischen Gesellschaft am 21. September 2011 in Leipzig
}

\section{Heinz Grohmann}

Online publiziert: 19. September 2012

(C) Springer 2012

Die Statistik nimmt heute auf weiten Gebieten des öffentlichen Lebens eine Achtung gebietende, einflussreiche Stellung ein. Bund, Länder und Gemeinden, Allgemeinheit, Wirtschaft, Wissenschaft bedienen sich ihrer Hilfe in ausgedehntem Maße. Die Statistik ist zu einer Wissenschaft geworden.

Das sind, leicht abgewandelt, die Worte, mit denen vor 100 Jahren eine große Statistikergemeinde in Deutschland ihrem Nestor Georg von Mayr eine Ehrengabe zu seinem 70. Geburtstag übereignete. ${ }^{1}$ Dieser Anlass wurde zum Startpunkt für die Gründung der Deutschen Statistischen Gesellschaft am 17. Juni 1911 in Dresden, interessanterweise als Abteilung der Deutschen Gesellschaft für Soziologie. Erster Vorsitzender wurde Georg von Mayr.

Wie hat sich die Welt doch seitdem verändert! Auch die Statistik ist längst nicht mehr nur die von 1911. Gleichwohl verdienten es die zitierten Worte, auch heute zu gelten, eher noch ausgedehnt auf weitere Lebensbereiche.

Die Entwicklung dieser 100 Jahre hat auch mein Leben geprägt. Ich bin ja nur 10 Jahre jünger als die Gesellschaft, wie sie in Dresden geboren, und als Statistiker mit Leib und Seele habe ich mich immer gefühlt.

Was war das Faszinierende, das die Gründer der Deutschen Statistischen Gesellschaft vor 100 Jahren und später mich zu solcher Euphorie veranlasste? Es war die Entdeckerfreude, mit den Mitteln der Statistik objektive Einsichten in die - wie von Mayr sagte - ,mannigfaltigen Gestaltungen und Gesetzmäßigkeiten des menschli-

${ }^{1}$ Zahn (1911) S V.

Zum gesamten Beitrag siehe Grohmann et al. (2011).

H. Grohmann ( $₫)$

Kronberg im Taunus, Deutschland

e-mail: heinz.grohmann@web.de 
chen gesellschaftlichen Lebens“ zu gewinnen, und die waren immer überaus spannend. Schon damals ging es um die Folgen eines als dramatisch empfundenen Geburtentrückgangs, um die soziale Lage der Menschen, um das Unterrichtswesen und vieles andere. In den folgenden Jahrzehnten erlangten gesamtwirtschaftliche Phänomene wie Volkseinkommen und Konjunktur, aber auch betriebswirtschaftliche das Interesse der Statistiker in Deutschland. Und diese genossen international ein hohes Ansehen. Der Vorsitzende der Gesellschaft nach dem Tode Georg von Mayrs, Friedrich Zahn, war zugleich Präsident des Internationalen Statistischen Instituts und wurde noch 10 Jahre später zum Ehrenpräsidenten gewählt.

Wohl gab es damals auch bedeutende mathematische Statistiker wie Wilhelm Lexis, Ladislaus von Bortkiewicz und Alexandr Tschuprow. Doch standen sie in der Deutschen Statistischen Gesellschaft eher am Rande, und von der englischen Schule der Statistik um Francis Galton und Karl Pearson nahmen nur die Wenigsten Notiz.

Nach Hitlers Machtergreifung suchten die amtlichen Statistiker als treue Staatsdiener ihre Aufgaben wie gewohnt zu erfüllen, wohl auch in der trügerischen Hoffnung, sich damit von der Politik fernhalten zu können. Doch mussten sie zusehen, wie ihre jüdischen und politisch missliebige Fachkollegen gedemütigt, aus ihren Ämtern entfernt wurden oder sich zum Verlassen des Landes gezwungen sahen. Ich erinnere nur an drei: Ernst Julius Gumbel, Richard von Mises und Eugen Würzburger, einen der Mitgründer der Deutschen Statistischen Gesellschaft. Unter den verbleibenden Statistikern gab es wie in der Bevölkerung auch alle Abstufungen im Verhalten zum Regime - von erzwungener Anpassung über bereitwilliges Mitläufertum bis zu bewusster Täterschaft. Ich weiß aus persönlichem Erleben - ich ging damals aufs Gymnasium und mein Vater war Beamter - welchen Demütigungen und Gefahren ein Staatsdiener ausgesetzt war, der sich dem System zu verweigern suchte. Ich habe aber auch erlebt, wie viele bereitwillig mitmachten. Der Vorsitzende Friedrich Zahn genoss das nun auch für Gesellschaften geltende Führerprinzip, war gegenüber Hitler geradezu devot und stellte diesen, was sein Verhältnis zur Statistik anbelangt, in eine Reihe mit Friedrich dem Großen, Napoleon und Mussolini. Die Bevölkerungsund Wirtschaftsstatistik blieb weiterhin dominant, nun nur durchsetzt mit Themen, die dem Regime dienlich waren. Einige der Rassenideologie nahestehende Bevölkerungsstatistiker schrieben damals freilich ein höchst unrühmliches Kapitel. Gravierend war aber auch die völlige Abschottung der Statistik von den Entwicklungen im Ausland.

Nach dem zweiten Weltkrieg wandelte sich das Bild nachhaltig. Es begann mit der Neugründung der Deutschen Statistischen Gesellschaft 1948 in München durch den Präsidenten des Bayerischen Statistischen Landesamtes Karl Wagner. Dieser erstrebte rasch einen Anschluss an die internationale Entwicklung und förderte innovative Methoden, wie den Einsatz von Stichprobenverfahren, auch in der amtlichen Statistik. Unser Kollege Heinrich Strecker hat das persönlich miterlebt, und Stichproben wurden sein bevorzugtes Forschungsgebiet.

In den folgenden Aufbaujahren nahm die amtliche Statistik in der Bundesrepublik einen ungeahnten Aufschwung. Nachfolger von Karl Wagner in der Deutschen Statistischen Gesellschaft wurde 1960 bezeichnenderweise der Präsident des Statistischen Bundesamtes Gerhard Fürst, der zusammen mit Hildegard Bartels, einer späteren Vorsitzenden, die Volkswirtschaftlichen Gesamtrechnungen für Deutschland aufbaute und die amtliche Statistik daran ausrichtete. Meine persönlichen Beziehungen 
zu Fürst und Bartels und zu meinem akademischen Lehrer Adolf Blind begründeten damals mein Engagement für die amtliche Statistik und für die Deutsche Statistische Gesellschaft. Mein erstes Forschungsgebiet war jedoch - die dynamische Rente war gerade eingeführt worden - die Frage nach deren langfristiger Finanzierbarkeit.

An den Universitäten nahm die Zahl der Lehrstühle für Statistik, vor allem in Wirtschafts- und Sozialwissenschaftlichen Fakultäten, deutlich zu; doch trat hier, orientiert an internationalen Vorbildern, die Methodenentwicklung ganz in den Vordergrund. In der Deutschen Statistischen Gesellschaft war es Wolfgang Wetzel, der als Vorsitzender ab 1972 den Einfluss der Universitäten und damit der Methodenforschung erheblich verstärkte und ihre Vertreter zu gleichrangigen Partnern der amtlichen und sonstigen angewandten Statistik machte. Damit endete deren bisherige Dominanz, und in vierjährigem Wechsel übernahm jeweils ein Hochschullehrer Ausnahme: Hildegard Bartels - den Vorsitz in der Gesellschaft. Beide Seiten gestalteten nunmehr gleichrangig das Geschehen in der Gesellschaft. Es bleibt Wetzels Verdienst, diese Neuorientierung durchgesetzt und dennoch den Zusammenhalt gewahrt zu haben.

Das Zusammenwirken beider Seiten im Vorstand war persönlich harmonisch und von wechselseitigem Respekt gekennzeichnet. Wir haben in dieser Zeit viele spannende Statistische Wochen erlebt, oft mit politisch hoch relevanten Themen, etwa zum Arbeitsmarkt, zur Umwelt und zur sozialen Sicherung. In diese Zeit fiel auch die Wiedervereinigung, nach der die Gesellschaft sehr bald Verbindung zu den Statistikern in den neuen Bundesländern aufnahm und Jahrestagungen in Berlin, Leipzig und Dresden sowie zahlreiche Pfingsttagungen in den neuen Bundesländern veranstaltete. Ein Höhepunkt war zudem die Ausrichtung der Tagung des Internationalen Statistischen Instituts 2003 in Berlin. Die fachliche Ausdifferenzierung erfolgte in den teils mehr praktisch, teils mehr theoretisch ausgerichteten Ausschüssen.

Inhaltlich gingen beide Seiten verschiedene Wege. Das Arbeitsfeld der Statistiker in den Ämtern, Unternehmen und Behörden blieb die Bevölkerungs-, Wirtschaftsund Sozialstatistik und damit weitgehend konzentriert auf nationale und europäische Aufgaben. An den Universitäten dominierte die Methode, konkret: die Stochastik, was zugleich eine enge Verbindung zur internationalen wissenschaftlichen Statistik sicherstellte.

Warum aber ergänzten sie sich nicht wechselseitig? Lassen Sie mich kurz einen Erklärungsversuch wagen. Statistik ist in allen empirischen Wissenschaften äußerst fruchtbar. Aber die Anwendungsbedingungen für die Stochastik sind nicht überall gleich. In Biologie, Medizin und Technik findet sie oftmals geradezu ideale Voraussetzungen. Auch in der Wirtschaftsforschung hat die Stochastik längst erfolgreich Verbreitung gefunden; doch führt sie hier allein nicht zum Ziel. In Wirtschaft und Gesellschaft bestimmt das von historischen, institutionellen und kulturellen Rahmenbedingungen abhängige, an Werten und Normen orientierte, vielfach interessengeleitete Verhalten der Menschen so weitgehend das Geschehen, dass schon eine sinnvolle Begriffsbildung und damit auch die Datenerhebung einen ganz eigenen, geradezu kulturorientierten Zugang erfordern. Das ist das Adäquationsproblem. ${ }^{2}$ Aber auch

${ }^{2}$ Grohmann (1988). 
die Datenanalyse und erst recht die Interpretation der Ergebnisse verlangen ein Mitbedenken des kulturellen Hintergrunds. Erwerbstätigkeit und Einkommen, Produktivität und Inflation, Wohlfahrt und Altersarmut sind anders weder sinnvoll zu messen noch adäquat zu analysieren und damit auch kaum tauglich für die Politikberatung. Zum formalen muss ein materielles Denken hinzutreten. Das aber ist charakteristisch für die praktische statistische Arbeit in den Ämtern, Unternehmen, Behörden und in der empirischen Wirtschaftsforschung. ,Zahl ohne Wort ist Zahlenmord“ sagte schon Siegmund Schott, einer der Mitgründer der Deutschen Statistischen Gesellschaft.

Inzwischen sind Theorie und Praxis schrittweise einander näher gekommen, und die Deutsche Statistische Gesellschaft hat das auch gebührend thematisiert. Die Arbeiten im Sonderforschungsbereich 3, aus dem das Sozioökonomische Panel hervorgegangen ist, waren ein frühes Beispiel. Auch die Erforschung der Arbeits- und der Finanzmärkte bezieht im Streben nach praktisch verwertbaren Ergebnissen die rechtlichen Regelungen und die menschlichen Triebkräfte in ihre Analysen ein. Als besonders fruchtbar erwies sich die Mikroökonometrie. Die amtliche Statistik unterstützt dies durch ihre Forschungsdatenzentren. Auch Zensus und Mikrozensus sind Beispiele verstärkter Kooperation zwischen amtlicher und mathematischer Statistik. Mit der Diskussion über Wohlfahrtsindikatoren jenseits des Bruttoinlandsprodukts, einem Hauptthema dieser Statistischen Woche, setzt die Gesellschaft diesen Weg eindrucksvoll fort.

So darf man im Rückblick auf die vergangenen 100 Jahre sagen: Die Statistik in Deutschland hat in dieser Zeit manchen Wandel erfahren. Die Deutsche Statistische Gesellschaft hat diese Entwicklung in all ihren Facetten nach Kräften gefördert, ihre Vertreter zusammengeführt und wieder Anschluss an die internationale Entwicklung gefunden. Sie wird auch in Zukunft ihrer Verantwortung für eine leistungsfähige, politisch unabhängige Statistik wahrnehmen. Wissenschaft und Gesellschaft bedürfen ihrer - künftig mehr denn je.

\section{Literatur}

Grohmann H (1988) Die statistische Adäquation als Postulat einer sachgerechten Abstimmung zwischen Theorie und Empirie. In: Freimann K-D, Ott AE (Hrsg) Theorie und Empirie in der Wirtschaftsforschung. Verlag J.C.B. Mohr, Tübingen

Grohmann H, Krämer W, Steger A (2011) Statistik in Deutschland - 100 Jahre Deutsche Statistische Gesellschaft. Springer, Berlin

Zahn F (Hrsg) (1911) Die Statistik in Deutschland nach ihrem heutigen Stand. Ehrengabe für Georg von Mayr. J. Schweizer Verlag, München 